\title{
EQUIVARIANT COMPRESSION OF CERTAIN DIRECT LIMIT GROUPS AND AMALGAMATED FREE PRODUCTS
}

\author{
CHRIS CAVE and DENNIS DREESEN \\ School of Mathematics, University of Southampton, Highfield, \\ Southampton, SO17 1BJ, United Kingdom \\ e-mails: chriscave89@gmail.com,Dennis.Dreesen@soton.ac.uk
}

(Received 19 June 2014; revised 22 June 2015; accepted 23 January 2016; first published online 10 June 2016)

\begin{abstract}
We give a means of estimating the equivariant compression of a group $G$ in terms of properties of open subgroups $G_{i} \subset G$ whose direct limit is $G$. Quantifying a result by Gal, we also study the behaviour of the equivariant compression under amalgamated free products $G_{1} *_{H} G_{2}$ where $H$ is of finite index in both $G_{1}$ and $G_{2}$.
\end{abstract}

2010 Mathematics Subject Classification. 20F65, 22D10.

1. Introduction. The Haagerup property, which is a strong converse of Kazhdan's property $(\mathrm{T})$, has translations and applications in various fields of mathematics such as representation theory, harmonic analysis, operator K-theory and so on. It implies the Baum-Connes conjecture and related Novikov conjecture [7]. We use the following definition of the Haagerup property.

DEFINITION 1.1. A locally compact second countable group $G$ is said to satisfy the Haagerup property if it admits a continuous proper affine isometric action $\alpha$ on some Hilbert space $\mathcal{H}$. Here, proper means that for every $M>0$, there exists a compact set $K \subset G$ such that $\|\alpha(g)(0)\| \geq M$ whenever $g \in G \backslash K$. We say that the action is continuous if the associated map $G \times \mathcal{H} \rightarrow \mathcal{H},(g, v) \mapsto \alpha(g)(v)$ is jointly continuous.

CONVENTION 1.2. Throughout this paper, all actions are assumed continuous and all groups will be second countable and locally compact.

Recall that any affine isometric action $\alpha$ can be written as $\pi+b$ where $\pi$ is a unitary representation of $G$ and where $b: G \rightarrow \mathcal{H}, g \mapsto \alpha(g)(0)$ satisfies

$$
\forall g, h \in G: b(g h)=\pi(g) b(h)+b(g) .
$$

In other words, $b$ is a 1-cocycle associated to $\pi$.

In [13], the authors define compression as a means to quantify how strongly a finitely generated group satisfies the Haagerup property. More generally, assume that $G$ is a compactly generated group. Denote by $S$ some compact generating subset and equip $G$ with the word length metric relative to $S$. Using the triangle inequality, one checks easily that any 1-cocycle $b$ associated to a unitary action of $G$ on a Hilbert space is Lipschitz. On the other hand, one can look for the supremum of $r \in[0,1]$ such that there exists $C, D>0$ with

$$
\forall g \in G: \frac{1}{C}|g|^{r}-D \leq\|b(g)\| \leq C|g|+D .
$$


DEFINITION 1.3. The above supremum, denoted $R(b)$, is called the compression of $b$ and taking the supremum over all proper affine isometric actions of $G$ on all Hilbert spaces leads to the equivariant Hilbert space compression of $G$, denoted $\alpha_{2}^{\#}(G)$. Suppose now that $G$ is no longer compactly generated but still has a proper length function. Then, define $\alpha_{2}^{\#}(G)$ to be the supremum of $R(b)$ but over all large-scale Lipschitz 1-cocycles.

The equivariant Hilbert space compression contains information on the group. First of all, if $\alpha_{2}^{\#}(G)>0$, then $G$ is Haagerup. The converse was disproved by T. Austin in [4], where the author proves the existence of finitely generated amenable groups with equivariant compression 0 . Further, it was shown in [13] that if for a finitely generated group $\alpha_{2}^{\#}(G)>1 / 2$, then $G$ is amenable. This result was generalized to compactly generated groups in [9] and it provides some sort of converse for the well-known fact that amenability implies the Haagerup property. Much effort has been done to calculate the explicit equivariant compression value of several groups and classes of groups, see e.g. $[2,5,12,19,20]$.

Given two finitely generated group $G$ and $H$ the group $\bigoplus_{H} G$ is no longer finitely generated. However, we can view $\bigoplus_{H} G$ as a subspace of $G$ ? $H$ and so equip $\bigoplus_{H} G$ with a natural proper metric. In this article, we are motivated by comparing the compression of $\bigoplus_{H} G$ with $G$ ? $H$. We assume that a given group $G$, equipped with a proper length function $l$, can be viewed as a direct limit of open (hence closed) subgroups $G_{1} \subset G_{2} \subset G_{3} \subset \ldots \subset G$. We equip each $G_{i}$ with the subspace metric from $G$. Our main objective will be to find bounds on $\alpha_{2}^{\#}(G)$ in terms of properties of the $G_{i}$. Note that, as each $G_{i}$ is a metric subspace of $G$, we have $\alpha_{2}^{\#}(G) \leq \inf _{i \in \mathbb{N}} \alpha_{2}^{\#}\left(G_{i}\right)$. The main challenge is to find a sensible lower bound on $\alpha_{2}^{\#}(G)$. The key property that we introduce is the $(\alpha, l, q)$ polynomial property, which we shorten to $(\alpha, l, q)$-PP (see Definition 2.5 below). Precisely, we obtain the following result.

THEOREM 1.4. Let $G$ be a locally compact, second countable group equipped with a proper length function l. Suppose there exists a sequence of open subgroups $\left(G_{i}\right)_{i \in \mathbb{N}}$, each equipped with the restriction of $l$ to $G_{i}$, such that $\lim _{i} G_{i}=G$ and $\alpha=\inf \left\{\alpha_{2}^{\#}\left(G_{i}\right)\right\}>0$. If $\left(G_{i}\right)_{i \in \mathbb{N}}$ has $(\alpha, l, q)-P P$, then there are the following two cases:

$$
l \geq q \Rightarrow \alpha_{2}^{\#}(G) \geq \frac{\alpha}{2 l+1}
$$

or,

$$
l \leq q \Rightarrow \alpha_{2}^{\#}(G) \geq \frac{\alpha}{l+q+1}
$$

We use this result to obtain a lower bound of the compression of the following examples. Let $F:[0,1] \times \mathbb{R}^{\geq 0} \rightarrow \mathbb{R}$ be the function

$$
F(\alpha, d)= \begin{cases}d(2 \alpha-1) & \text { if } 2 \alpha \geq 1 \\ 0 & \text { otherwise }\end{cases}
$$


THEOREM 1.5. Let $G$ and $H$ be finitely generated groups where $H$ has polynomial growth of degree $d \geq 1$. Then,

$$
\alpha_{2}^{\#}\left(\bigoplus_{H} G\right) \geq \frac{\alpha_{2}^{\#}(G)}{1+F\left(\alpha_{2}^{\#}(G), d\right)+2 \alpha_{2}^{\#}(G)(1+d)},
$$

where $\bigoplus_{H} G$ is equipped with the subspace metric from $G$ ? $H$.

Our result also allows to consider spaces $\bigoplus_{H} G_{h}$ where $G_{h}$ actually depends on the parameter $h \in H$. For example, we take a collection of finite groups $F_{i}$ with $F_{0}=\{0\}$ and look at $G=\bigoplus_{i \in \mathbb{N}} F_{i}$. This is the first available lower bound for the equivariant compression of groups of this type.

THEOREM 1.6. Let $\left\{F_{i}\right\}_{i \in \mathbb{N}}$ be a collection of finite groups. Equip $G=\bigoplus_{i \in \mathbb{N}} F_{i}$ with the length function $l(g)=\min \left\{n \in \mathbb{N}: g \in \bigoplus_{i=0}^{n} F_{i}\right\}$. Then, $\alpha_{2}^{\#}(G, l)>1 / 3$.

We give a proof of Theorem 1.4 in Section 2.2 and apply to these concrete examples in Section 2.3. Note that our result can also be viewed as a study of the behaviour of equivariant compression under direct limits. The behaviour of the Haagerup property and the equivariant compression under group constructions has been studied extensively (see e.g. $[\mathbf{1 1}, \mathbf{1 8}]$, Chapter 6 of $[\mathbf{1}, \mathbf{7}, \mathbf{8}]$ ).

In Section 3, we quantify part of [12] to study the behaviour of the equivariant compression under certain amalgamated free products $G_{1} *_{H} G_{2}$ where $H$ is of finite index in both $G_{1}$ and $G_{2}$. Suppose $H$ is a closed finite index subgroup inside groups compactly generated groups $G_{1}$ and $G_{2}$ and there exists proper affine isometric actions $\beta_{i}: G_{i} \rightarrow \operatorname{Aff}\left(V_{i}\right)$ on Hilbert spaces $V_{i}$. In [12], the author shows that if there exists a non-trivial closed subspace $W \subset V_{1} \cap V_{2}$ that is fixed by the restricted actions $\left.\beta_{i}\right|_{H}$ then the product $G_{1} *_{H} G_{2}$ also admits a proper affine isometric action on a Hilbert space. We quantify this result.

THEOREM 1.7. With the above assumptions $\alpha_{2}^{\#}\left(G_{1} *_{H} G_{2}\right) \geq \frac{\alpha_{2}^{\#}(H)}{2}$

\section{The equivariant compression of direct limits of groups}

2.1. Preliminaries and formulation of the main result. Suppose $G$ is a locally compact second countable group equipped with a proper length function $l$, i.e. closed $l$-balls are compact. Assume that there exists a sequence of open subgroups $G_{i} \subset G$ such that $\lim _{\longrightarrow} G_{i}=G$, i.e. $G$ is the direct limit of the $G_{i}$. We equip each $G_{i}$ with the restriction of $l$ to $G_{i}$. It will be our goal to find bounds on $\alpha_{2}^{\#}(G)$ in terms of the $\alpha_{2}^{\#}\left(G_{i}\right)$. Clearly, as the $G_{i}$ are subgroups then an upper bound of the equivariant compression is the infimum of the equivariant compressions of the $G_{i}$. The challenge is to find a sensible lower bound. The next example will show that it is not enough to only consider the $\alpha_{2}^{\#}\left(G_{i}\right)$.

EXAMPLE 2.1. Consider the wreath product $\mathbb{Z} \imath \mathbb{Z}$ equipped with the standard word metric relative to $\left\{\left(\delta_{1}, 0\right),(0,1)\right\}$, where $\delta_{1}$ is the characteristic function of $\{0\}$. Let $\mathbb{Z}^{(\mathbb{Z})}=\{f: \mathbb{Z} \rightarrow \mathbb{Z}: f$ is has finite support $\}$ be equipped with the subspace metric from $\mathbb{Z} \imath \mathbb{Z}$. Consider the direct limit of groups

$$
\mathbb{Z} \hookrightarrow \mathbb{Z}^{3} \hookrightarrow \mathbb{Z}^{5} \cdots \hookrightarrow \mathbb{Z}^{(\mathbb{Z})}
$$


where $\mathbb{Z}^{2 n+1}$ has the subspace metric from $\mathbb{Z}^{(\mathbb{Z})}$. This metric is quasi-isometric to the standard word metric on $\mathbb{Z}^{2 n+1}$ and so each term has equivariant compression 1 . So $\mathbb{Z}^{(\mathbb{Z})}$ is a direct limit of groups with equivariant compression 1 but by [2] has equivariant compression less than $3 / 4$. On the other hand the sequence

$$
\mathbb{Z} \rightarrow \mathbb{Z} \rightarrow \cdots \rightarrow \mathbb{Z}
$$

is a sequence of groups with equivariant compression 1 and the equivariant compression of the direct limit is 1 .

Given a sequence of 1-cocycles $b_{i}$ of $G_{i}$, then in order to predict the equivariant compression of the direct limit, it will be necessary to incorporate more information on the growth behaviour of the $b_{i}$ than merely the compression exponent $R\left(b_{i}\right)$. The growth behaviour of 1-cocycles can be completely caught by so called conditionally negative definite functions on the group (See Proposition 2.3 and Theorem 2.4 below).

Definition 2.2. A continuous map $\psi: G \rightarrow \mathbb{R}^{+}$is called conditionally negative definite if $\psi(g)=\psi\left(g^{-1}\right)$ for every $g \in G$ and if for all $n \in \mathbb{N}, \forall g_{1}, g_{2}, \ldots, g_{n} \in G$ and all $a_{1}, a_{2}, \ldots, a_{n} \in \mathbb{R}$ with $\sum_{i=1}^{n} a_{i}=0$, we have

$$
\sum_{i, j} a_{i} a_{j} \psi\left(g_{i}^{-1} g_{j}\right) \leq 0
$$

Proposition 2.3 (Example 13, page 62 of [10]). Let $\mathcal{H}$ be a Hilbert space and $b: G \rightarrow \mathcal{H}$ a l-cocycle associated to a unitary representation. Then, the map $\psi: G \rightarrow \mathbb{R}$, $g \mapsto\|b(g)\|^{2}$ is a conditionally negative definite function on $G$.

TheOREM 2.4 (Proposition 14, page 63 of [10]). Let $\psi: G \rightarrow \mathbb{R}$ be a conditionally negative definite function on a group $G$. Then, there exists an affine isometric action $\alpha$ on a Hilbert space $\mathcal{H}$ such that the associated 1-cocycle satisfies $\psi(g)=\|b(g)\|^{2}$.

These two results imply that we can pass between conditionally negative definite functions and 1-cocycles associated to unitary actions.

DefinITION 2.5. Let $G$ be a group equipped with a proper length function $l$ and suppose that $\left(G_{i}\right)_{i \in \mathbb{N}}$ is a normalized nested sequence of open subgroups such that $\lim G_{i}=G$. Assume that $\alpha:=\inf _{i \in \mathbb{N}} \alpha_{2}^{\#}\left(G_{i}\right) \in(0,1]$ and $l, q \geq 0$. The sequence $\left(G_{i}\right)_{i}$ $\overrightarrow{\text { has }}$ the $(\alpha, l, q)$-polynomial property $((\alpha, l, q)$-PP) if there exists:

(1) a sequence $\left(\eta_{i}\right)_{i} \subset \mathbb{R}^{+}$converging to 0 such that $\eta_{i}<\alpha$ for each $i \in \mathbb{N}$,

(2) $\left(A_{i}, B_{i}\right)_{i \in \mathbb{N}} \subset \mathbb{R}^{>0} \times \mathbb{R}^{\geq 0}$,

(3) a sequence of 1-cocycles $\left(b_{i}: G_{i} \rightarrow \mathcal{H}_{i}\right)_{i \in \mathbb{N}}$, where each $b_{i}$ is associated to a unitary action $\pi_{i}$ of $G_{i}$ on a Hilbert space $\mathcal{H}_{i}$

such that

$$
\frac{1}{A_{i}}|g|^{2 \alpha-\eta_{i}}-B_{i} \leq\left\|b_{i}(g)\right\|^{2} \leq A_{i}|g|^{2}+B_{i} \quad \forall g \in G_{i}, \forall i \in \mathbb{N}
$$

and there is $C, D>0$ such that $A_{i} \leq C i^{l}, B_{i} \leq D i^{q}$ for all $i \in \mathbb{N}$.

Note that the only real restrictions are the inequalities $A_{i} \leq C i^{l}, B_{i} \leq D i^{q}$ : we exclude sequences $A_{i}, B_{i}$ that grow faster than any polynomial. The intuition is that equivariant compression is a polynomial property (this follows immediately from its 
definition), so that sequences $A_{i}, B_{i}$ growing faster than any polynomial would be too dominant and one would lose all hope of obtaining a lower bound on $\alpha_{2}^{\#}(G)$. On the other hand, if the $A_{i}$ and $B_{i}$ grow polynomially, then one can use compression to somehow compensate for this growth. One then obtains a strictly positive lower bound on $\alpha_{2}^{\#}(G)$ which may decrease depending on how big $l$ and $q$ are. We have the following useful characterisation of $(\alpha, l, q)$-polynomial property.

LEMMA 2.6. Let $G$ be a locally compact second countable group and $l$ is a proper length metric. Suppose there exists a sequence of open subgroups $\left(G_{i}\right)_{i \in \mathbb{N}}$ such that $\lim _{i} G_{i}=G$. If each $G_{i}$ are equipped with the restricted length metric from $G$ then $\left(G_{i}\right)_{i \in \mathbb{N}}$ $\overrightarrow{h a s}$ the $(\alpha, l, q)$-polynomical property if and only if there exists $C, D>0$ such that for all $\varepsilon>0$ there exists

(1) a sequence $\left(A_{i}, B_{i}\right)_{i \in \mathbb{N}} \subset \mathbb{R}^{>0} \times \mathbb{R}^{\geq 0}$ such that $A_{i} \leq C i^{l}$ and $B_{i} \leq D i^{q}$;

(2) a sequence of 1-cocycles $\left(b_{i}: G_{i} \rightarrow \mathcal{H}_{i}\right)_{i \in \mathbb{N}}$

such that

$$
\frac{1}{A_{i}}|g|^{2 \alpha-\varepsilon}-B_{i} \leq\left\|b_{i}(g)\right\|^{2} \leq A_{i}|g|^{2}+B_{i} \quad \forall g \in G_{i}, \forall i \in \mathbb{N}
$$

Proof. The "if" direction is obvious. For the "only if" direction fix $\varepsilon>0$ and suppose $\left(G_{i}\right)_{i \in \mathbb{N}}$ has the $(\alpha, l, q)$-polynomial property with respect to sequences $\left(\eta_{i}\right)_{i \in \mathbb{N}}$ and $\left(b_{i}: G_{i} \rightarrow \mathcal{H}_{i}\right)_{i \in \mathbb{N}}$. Choose $N \in \mathbb{N}$ large enough so that $\eta_{k}<\varepsilon$ for all $k \geq N$. Thus, $b_{k}: G_{k} \rightarrow \mathcal{H}_{k}$ satisfies the above conditions for all $k \geq N$. For $k \leq N$ we take the restriction of $b_{N}$ to $G_{k}$ to obtain the sequence satisfying the above conditions for all $k \in \mathbb{N}$.

Proposition 2.7. Let $G$ be a locally compact second countable group and suppose there exists a sequence of open subgroups $\left(G_{i}\right)_{i \in \mathbb{N}}$ such that $\lim _{\rightarrow} G_{i}=G$. If $\alpha:=\alpha_{2}^{\#}(G)>0$ then $\left(G_{i}\right)_{i \in \mathbb{N}}$ has $(\alpha, 0,0)$-polynomical property.

Proof. For all $0<\varepsilon<\alpha$ there exists a 1 -cocycle $b$ such that

$$
\frac{1}{A}|g|^{\alpha-\varepsilon}-B \leq\|b(g)\| \quad \forall g \in G
$$

The restriction of $b$ to each $G_{i}$ is a 1 -cocycle and gives $\left(G_{i}\right)_{i \in \mathbb{N}}$ the $(\alpha, 0,0)$-polynomial property.

Combining this with Theorem 1.4 we have the following consequence which confirms our intuition.

COROLLARY 2.8. Let $G$ be a locally compact second countable group with a proper length function l. If there exists a sequence of open subgroups $\left(G_{i}\right)_{i \in \mathbb{N}}$ such that $\lim G_{i}=G$ then $\left(G_{i}\right)_{i \in \mathbb{N}}$ has the $(\alpha, l, q)$-polynomial property for some $\alpha \in(0,1]$ and $l, q \geq 0$ if and only if $\alpha_{2}^{\#}(G)>0$.

\subsection{The proof of Theorem 1.4}

Proof of Theorem 1.4. First, we can assume that $l$ is uniformly discrete. That is there exists a $c>0$ such that $l(x)>c$ for all $x \in G \backslash\{e\}$. This is because given a length function $l$ one can define a new length function $l^{\prime}$ such that $l^{\prime}(x)=1$ whenever 
$0<l(x) \leq 1$ and $l^{\prime}(x)=l(x)$ when $l(x) \geq 1$. Hence $l^{\prime}$ will be quasi-isometric to $l$ and so will not change the compression of $G$ or $G_{i}$.

Take sequences $\left(\psi_{i}: G_{i} \rightarrow \mathbb{R}\right)_{i \in \mathbb{N}}, \quad\left(\eta_{i}\right)_{i}$ and $(A, B)=\left(A_{i}, B_{i}\right)_{i \in \mathbb{N}} \subset \mathbb{R}^{>0} \times \mathbb{R}^{\geq 0}$ satisfying the conditions of $(\alpha, l, q)$-PP (see Definition 2.5). We assume here, without loss of generality, that the sequences $\left(A_{i}\right)_{i},\left(B_{i}\right)_{i}$ are non-decreasing.

For each $G_{i}$, define a sequence of maps $\left(\varphi_{k}^{i}: G_{i} \rightarrow \mathbb{R}\right)_{k \in \mathbb{N}}$ by

$$
\varphi_{k}^{i}(g)= \begin{cases}\exp \left(\frac{-\psi_{i}(g)}{k}\right) & \text { if } g \in G_{i} \\ 0 & \text { otherwise. }\end{cases}
$$

Note that each $\varphi_{k}^{i}$ is continuous as $G_{i}$ is open and also closed, being the complement of $\cup_{g \notin G_{i}} g G_{i}$. By $(\alpha, l, q)$-PP, for all $i, k \in \mathbb{N}$, we have

$$
\begin{aligned}
& \exp \left(\frac{-A_{i}|g|^{2}-B_{i}}{k}\right) \leq \varphi_{k}^{i}(g) \quad \forall g \in G_{i}, \text { and } \\
& \varphi_{k}^{i}(g) \leq \exp \left(\frac{-|g|^{2 \alpha-\eta_{i}}+A_{i} B_{i}}{A_{i} k}\right) \quad \forall g \in G .
\end{aligned}
$$

Fix some $p>0$, set $J(i)=\left(A_{i}+B_{i}\right) i^{1+p}$ and define $\bar{\psi}: G \rightarrow \mathbb{R}$ by

$$
\bar{\psi}(g)=\sum_{i \in \mathbb{N}} 1-\Phi_{i}(g)
$$

where $\Phi_{i}(g):=\varphi_{J(i)}^{i}(g)$. To check that $\bar{\psi}$ is well defined, choose any $g \in G$ and note that for $i>|g|$, we have $g \in G_{i}$ and so $\varphi_{k}^{i}(g) \geq \exp \left(\frac{-A_{i}|g|^{2}-B_{i}}{k}\right)$. Hence

$$
\begin{aligned}
\sum_{i>|g|} 1-\Phi_{i}(g) & \leq \sum_{i>|g|} 1-\exp \left(\frac{-A_{i}|g|^{2}-B_{i}}{\left(A_{i}+B_{i}\right) i^{1+p}}\right) \\
& \leq \sum_{i>|g|} 1-\exp \left(\frac{-|g|^{2}}{i^{1+p}}\right) \\
& \leq \sum_{i>|g|} \frac{|g|^{2}}{i^{1+p}}=|g|^{2} \sum_{i>|g|} \frac{1}{i^{1+p}}
\end{aligned}
$$

As

$$
\bar{\psi}(g)=\sum_{i=1}^{|g|} 1-\Phi_{i}(g)+\sum_{i>|g|} 1-\Phi_{i}(g)
$$

we see that $\bar{\psi}$ is well defined and that it can be written as a limit of continuous functions converging uniformly over compact sets. Consequently, it is itself continuous. By Schoenberg's theorem (see [10, Theorem 5.16]), all of the maps $\varphi_{k}^{i}$ are positive definite on $G_{i}$ and hence on $G$ (see [15, Section 32.43(a)]). In other words,

$$
\forall n \in \mathbb{N}, \forall a_{1}, a_{2}, \ldots, a_{n} \in \mathbb{R}, \forall g_{1}, g_{2}, \ldots, g_{n} \in G: \sum_{i, j=1}^{n} a_{i} a_{j} \varphi_{k}^{i}\left(g_{i}^{-1} g_{j}\right) \geq 0
$$


Hence, $\bar{\psi}$ is a conditionally negative definite map. Moreover, using that $l$ is uniformly discrete, we can find a constant $E>0$ such that

$$
\bar{\psi}(g) \leq|g|+|g|^{2} \sum_{i>|g|} \frac{1}{i^{1+p}} \leq E|g|^{2},
$$

so the 1-cocycle associated to $\bar{\psi}$ via Theorem 2.4 is large-scale Lipschitz.

Let us now try to find the compression of this 1-cocycle. Set $V I: \mathbb{N} \rightarrow \mathbb{R}$ to be the function

$$
V I(i)=\left(A_{i} J(i) \ln (2)+A_{i} B_{i}\right)^{\frac{1}{2 \alpha-\eta_{i}}} .
$$

One checks easily that

$$
|g| \geq V I(i) \Rightarrow \Phi_{i}(g)=\varphi_{J(i)}^{i}(g) \leq \frac{1}{2} .
$$

To make the function $V I$ more concrete, let us look at the values of $A_{i}, B_{i}$ and $J(i)$. Recall that by assumption, we have $A_{i} \leq C i^{l}, B_{i} \leq D i^{q}$. Hence for $i$ sufficiently large, we have $J(i) \leq\left(C i^{l}+D i^{q}\right) i^{1+p} \leq F i^{X}$ where $F$ is some constant and $X=$ $1+p+\max (l, q)$. We thus obtain that there is a constant $K>0$ such that for every $i$ sufficiently large (say $i>I$ for some $I \in \mathbb{N}_{0}$ ),

$$
V I(i) \leq K i^{Y /\left(2 \alpha-\eta_{i}\right)}
$$

where

$$
\begin{aligned}
Y & =\max (X+l, l+q), \\
& =\max (1+p+2 l, 1+p+l+q) .
\end{aligned}
$$

As the sequence $\eta_{i}$ converges to 0 , we can choose any $\delta>0$ and take $I>0$ such that in addition $\eta_{i}<\delta$ for $i>I$. We then have for all $i>I$ that

$$
V I(i) \leq K i^{Y /(2 \alpha-\delta)} .
$$

Together with equation (3), this implies that for $i>I$,

$$
|g| \geq K i^{Y /(2 \alpha-\delta)} \Rightarrow \Phi_{i}(g)=\varphi_{J(i)}^{i}(g) \leq \frac{1}{2}
$$

For every $g \in G$, set

$$
c(g)_{p, \delta}=\sup \left\{i \in \mathbb{N}\left|K i^{Y /(2 \alpha-\delta)} \leq\right| g \mid\right\} .
$$

We then have for every $g \in G$ with $|g|$ large enough, that

$$
\begin{aligned}
\bar{\psi}(g) & \geq \sum_{i=1}^{c(g)_{p, \delta}} 1-\varphi_{J(i)}^{i}(g), \\
& \geq \sum_{i=I+1}^{c(g)_{p, \delta}} 1 / 2=\frac{c(g)_{p, \delta}-I}{2} .
\end{aligned}
$$


As $c(g)_{p, \delta} \geq\left(\frac{|g|}{K}\right)^{(2 \alpha-\delta) / Y}-1$, we conclude that $R(b) \geq \frac{2 \alpha-\delta}{2 \max (1+p+2 l, 1+p+l+q)}$. As this is true for any small $p, \delta>0$, we can take the limit for $p, \delta \rightarrow 0$ to obtain $\alpha_{2}^{\#}(G) \geq$ $\frac{\alpha}{\max (1+2 l, 1+l+q)}$. Hence, we have the following two cases:

$$
l \geq q \Rightarrow \alpha_{2}^{\#}(G) \geq \frac{\alpha}{1+2 l}
$$

or,

$$
l \leq q \Rightarrow \alpha_{2}^{\#}(G) \geq \frac{\alpha}{l+q+1}
$$

\subsection{Examples}

Let $F:[0,1] \times \mathbb{R}^{\geq 0} \rightarrow \mathbb{R}$ be the function

$$
F(\alpha, d)= \begin{cases}d(2 \alpha-1) & \text { if } 2 \alpha \geq 1 \\ 0 & \text { otherwise }\end{cases}
$$

THEOREM 2.9. Let $G$ and $H$ be finitely generated groups where $H$ has polynomial growth of degree $d \geq 1$. Then,

$$
\alpha_{2}^{\#}\left(\bigoplus_{H} G\right) \geq \frac{\alpha_{2}^{\#}(G)}{1+F\left(\alpha_{2}^{\#}(G), d\right)+2 \alpha_{2}^{\#}(G)(1+d)},
$$

where $\bigoplus_{H} G$ is equipped with the subspace metric from $G$ ? $H$.

REMARK 2.10. Theorem 1.3. from [17] provides a lower bound to the compression of $G$ ? $H$. Under the assumptions in Theorem 2.9, Theorem 1.3. in [17] gives a lower bound $\alpha_{2}^{\#}(G, H) \geq \alpha_{1}^{\#}(G) / 2$. As this bound is in terms of $L^{1}$-compression, this makes comparison between the bound in Theorem 2.9 and [17, Theorem 1.3.] difficult. However, it is known that $\alpha_{2}^{\#}(G) \leq \alpha_{1}^{\#}(G) \leq 2 \alpha_{2}^{\#}(G)$ for all finitely generated groups $G$, see the proof of Theorem 1.1. and Theorem 1.3. in [17] and [18, Lemma 2.3.].

We use this to show that under some circumstances the above lower bound is larger than the bound provided in [17, Theorem 1.3.]. Suppose that $\alpha_{1}^{\#}(G) / 2<\alpha_{2}^{\#}(G)$. Then, there exists a $c>0$ such that $\frac{2 \alpha_{2}^{\#}(G)}{\alpha_{1}^{\#}(G)}>1+c$. If $\alpha_{2}^{\#}(G) \leq \min \left\{\frac{c}{2(1+d)}, 1 / 2\right\}$ then by Theorem 2.9

$$
\alpha_{2}^{\#}\left(\oplus_{H} G\right) \geq \frac{\alpha_{2}^{\#}(G)}{1+c}>\frac{\alpha_{1}^{\#}(G)}{2} .
$$

Unfortunately, the values of $\alpha_{2}^{\#}$ are not so well understood and at the time of writing the only know values for $\alpha_{2}^{\#}$ are $1,1 / 2,0$ and $\frac{1}{2-2^{1-k}}$ for $k \in \mathbb{N}[\mathbf{2}, \mathbf{4}, \mathbf{1 8}]$. In the nonequivariant case any value for compression can be achieved [3]. It is likely that there exists groups such that $\alpha_{2}^{\#}$ takes values strictly between 0 and $1 / 2$ in which case our theorem can be applied to provide larger lower bounds than $\alpha_{1}^{\#}(G) / 2$.

Proof. We consider $\bigoplus_{H} G$ to be the group of functions $\mathbf{f}: H \rightarrow G$ that have finite support. Let $\mathbf{f} \in \bigoplus_{H} G$ and let $\operatorname{Supp}(\mathbf{f})=\left\{h_{1}, \ldots, h_{n}\right\} \subset H$. Set the length of $\mathbf{f}$ as 
follows

$$
\begin{aligned}
|\mathbf{f}|_{G L}= & \inf _{\sigma \in S_{n}}\left(d_{H}\left(1, h_{\sigma(1)}\right)+\sum_{i=1}^{n} d_{H}\left(h_{\sigma(i)}, h_{\sigma(i+1)}\right)+d_{H}\left(h_{\sigma(n)}, 1\right)\right) \\
& +\sum_{h \in H}|\mathbf{f}(h)|_{G} .
\end{aligned}
$$

This is the induced length metric from $G$ ? $H$ and so this is a proper length function on $\bigoplus_{H} G$. Consider the following group

$$
G_{i}=\{\mathbf{f}: H \rightarrow G: \operatorname{Supp}(\mathbf{f}) \subset B(1, i)\}
$$

and set $n_{i}=|B(1, i)|$. Each $G_{i}$ is finitely generated and the restricted wreath metric to $G_{i}$ is proper and left invariant so the wreath metric and the word metric are quasiisometric. In particular

$$
|\mathbf{f}|_{G i H}-2 i|B(1, i)| \leq \sum_{h \in B(1, i)}|\mathbf{f}(h)|_{G} \leq|\mathbf{f}|_{G i H}
$$

for all $\mathbf{f} \in G_{i}$. By [14, Proposition 4.1. and Corollary 2.13.] it follows that $\alpha_{2}^{\#}\left(G_{i}\right)=$ $\alpha_{2}^{\#}(G)$ for all $i \in \mathbb{N}$. Set $0<\alpha<\alpha_{2}^{\#}(G)$ and consider a 1 -cocyle $b: G \rightarrow \mathcal{H}$ such that

$$
\frac{1}{C}|g|_{G}^{2 \alpha} \leq\|b(g)\|^{2} \leq C|g|_{G}^{2}
$$

Enumerate $B(1, i)$ so that $\left\{h_{1}, \ldots, h_{n_{i}}\right\}=B(1, i)$ and define a 1 -cocycle $b_{i}: G_{i} \rightarrow \mathcal{H}^{n_{i}}$, where $b_{i}(\mathbf{f})=\left(b\left(\mathbf{f}\left(h_{1}\right)\right), \ldots, b\left(\mathbf{f}\left(h_{n_{i}}\right)\right)\right)$. If $|\mathbf{f}|_{G i H}>4 i|B(1, i)|$, then

$$
\begin{aligned}
\left\|b_{i}(\mathbf{f})\right\|_{1 / \alpha}=\left(\sum_{j=1}^{i}\left\|b\left(\mathbf{f}\left(h_{n_{j}}\right)\right)\right\|^{1 / \alpha}\right)^{\alpha} & \geq \frac{1}{C^{1 / \alpha}}\left(\sum_{j=1}^{i}\left|\mathbf{f}\left(h_{n_{j}}\right)\right|_{G}\right)^{\alpha} \\
& \geq \frac{1}{C^{1 / \alpha}}\left(|\mathbf{f}|_{G i H}-2 i|B(1, i)|\right)^{\alpha} \geq \frac{1}{2 C^{1 / \alpha}}|\mathbf{f}|_{G i H}^{\alpha} .
\end{aligned}
$$

If $2 \alpha<1$ then $\left\|b_{i}(\mathbf{f})\right\|_{2} \geq\left\|b_{i}(\mathbf{f})\right\|_{1 / \alpha}$ for all $\mathbf{f} \in G_{i}$ and so it follows that

$$
\frac{1}{4 C^{2 / \alpha}}|\mathbf{f}|_{G 2 H}^{2 \alpha}-\frac{i^{2 \alpha}}{C}|B(1, i)|^{2 \alpha} \leq\left\|b_{i}(\mathbf{f})\right\|_{2}^{2},
$$

for all $\mathbf{f} \in G_{i}$. Hence $\left(G_{i}\right)_{i \in \mathbb{N}}$ has the $(\alpha, 0,2 \alpha(1+d))$ polynomial property. 
If $2 \alpha \geq 1$ then by Hölder's inequality $\left\|b_{i}(\mathbf{f})\right\|_{2} \geq n_{i}^{\frac{1-2 \alpha}{2}}\left\|b_{i}(\mathbf{f})\right\|_{1 / \alpha}$ for all $\mathbf{f} \in G_{i}$ and so it follows that

$$
\frac{1}{4 C^{2 / \alpha}|B(1, i)|^{2 \alpha-1}}|\mathbf{f}|_{G i H}^{2 \alpha}-\frac{i^{2 \alpha}}{C}|B(1, i)|^{2 \alpha} \leq\left\|b_{i}(\mathbf{f})\right\|_{2}^{2} .
$$

for all $\mathbf{f} \in G_{i}$. Hence $\left(G_{i}\right)_{i \in \mathbb{N}}$ has the $(\alpha, d(2 \alpha-1), 2 \alpha(1+d))$ polynomial property. Thus by Theorem 1.4 and that $\alpha, d \geq 0$ it follows that

$$
\alpha_{2}^{\#}\left(\bigoplus_{H} G\right) \geq \frac{\alpha}{1+F(\alpha, d)+2 \alpha(1+d)},
$$

for all $\alpha<\alpha_{2}^{\#}(G)$ and so the statement of the theorem holds.

THEOREM 2.11. Let $\left\{F_{i}\right\}_{i \in \mathbb{N}}$ be a collection of finite groups such that $F_{0}=\{1\}$. Let $G=\bigoplus_{i \in \mathbb{N}} F_{i}$ be equip with the proper length function $l(g)=\min \left\{n \in \mathbb{N}: g \in \bigoplus_{i=0}^{n} F_{i}\right\}$. Then $\alpha_{2}^{\#}(G) \geq 1 / 3$.

Proof. Set $G_{i}=\bigoplus_{j=0}^{i} F_{j}$ and observe that $\alpha_{2}^{\#}\left(G_{i}\right)=1$ as $G_{i}$ is finite for all $i \in \mathbb{N}$. Define $f_{i}: G_{i} \rightarrow \mathbb{R}$ to be the 0 -map. This is clearly a 1 -cocycle and satisfies

$$
\forall g \in G_{i}: l(g)^{2}-i^{2} \leq\left|f_{i}(g)\right|^{2} \leq l(g)^{2}+i^{2} .
$$

Hence $\left(G_{i}\right)_{i \in \mathbb{N}}$ has the (1,0,2)-polynomial property. Thus $\alpha_{2}^{\#}(G) \geq 1 / 3$.

EXAMPLE 2.12. We will use [3] to provide an example of a sequence that does not have $(\alpha, l, q)$-polynomial property for any $\alpha \in(0,1]$ and $l, q>0$. Let $\Pi_{k}, k \geq 1$ be a sequence of Lafforgue expanders that do not embed into any uniformly convex Banach space [16]. These are finite factor groups $M_{k}$ of a lattice $\Gamma$ of $\mathrm{SL}_{3}(F)$ for a local field $F$.

For every $\alpha \in[0,1]$ there exists a finitely generated group $G$ and a sequence of scaling constants $\lambda_{k}$ such that $\lambda_{k} \Pi_{k}$ has compression $\alpha$ and $G$ is quasi-isometric to $\lambda_{k} \Pi_{k}$. Furthermore, $G$ contains the free product $*_{k} M_{k}$ as a subgroup. Let $\alpha=0$ and let $G$ and the scaling constants $\lambda_{k}$ be such that $G$ has compression 0 . We can equip $*_{k} M_{k}$ with a proper left invariant metric coming from $G$. Hence we have a sequence

$$
M_{1} \hookrightarrow M_{1} * M_{2} \hookrightarrow \cdots \hookrightarrow *_{k=1}^{n} M_{k} \hookrightarrow \cdots \hookrightarrow *_{k} M_{k}
$$

For each $n>0, *_{k=1}^{n} M_{k}$ has equivariant compression $1 / 2$ [11, Theorem 1.4.] however the limit group $*_{k} M_{k}$ contains a quasi-isometric copy of $\lambda_{k} \Pi_{k}$ and so has compression 0 . Thus, this sequence cannot have the $(\alpha, l, q)$-polynomial property for any $\alpha \in(0,1]$ and $l, q>0$.

3. The behaviour of compression under free products amalgamated over finite index subgroups. It is known that the Haagerup property is not preserved under amalgamated free products. Indeed, $\left(S L_{2}(\mathbb{Z}) \rtimes \mathbb{Z}^{2}, \mathbb{Z}^{2}\right)$ has the relative property $(T)$. So $S L_{2}(\mathbb{Z}) \rtimes \mathbb{Z}^{2}=\left(\mathbb{Z}_{6} \rtimes \mathbb{Z}^{2}\right) *\left(\mathbb{Z}_{2} \rtimes \mathbb{Z}^{2}\right)\left(\mathbb{Z}_{4} \rtimes \mathbb{Z}^{2}\right)$ is not Haagerup. In [12], S.R. Gal proves the following result.

THEOREM 3.1. Let $G_{1}$ and $G_{2}$ be finitely generated groups with the Haagerup property that have a common finite index subgroup $H$. For each $i=1,2$, let $\beta_{i}$ be a proper affine isometric action of $G_{i}$ on a Hilbert space $V_{i}\left(=l^{2}(\mathbb{Z})\right)$. Assume that $W<V_{1} \cap V_{2}$ is 
invariant under the actions $\left(\beta_{i}\right)_{\mid H}$ and moreover that both these (restricted) actions coincide on $W$. Then, $G_{1} *_{H} G_{2}$ is Haagerup.

Under the same conditions as above, we want to give estimates on $\alpha_{2}^{\#}\left(G_{1} *_{H} G_{2}\right)$ in terms of the equivariant Hilbert space compressions of $G_{1}, G_{2}$ (see Theorem 3.3 below). Note that the following lemma shows that $\alpha_{2}^{\#}\left(G_{1}\right)=\alpha_{2}^{\#}(H)=\alpha_{2}^{\#}\left(G_{2}\right)$ when $H$ is of finite index in both $G_{1}$ and $G_{2}$. We are indebted to Alain Valette for this lemma and its proof. The notation $\alpha_{p}^{\#}$ refers to the equivariant $L_{p}$-compression for some $p \geq 1$. It is defined in exactly the same way as $\alpha_{2}^{\#}$ except that one considers affine isometric actions on $L_{p}$-spaces instead of $L_{2}$-spaces.

LEMMA 3.2. Let $G$ be a compactly generated, locally compact group, and let $H$ be an open, finite-index subgroup of $G$. Then, $\alpha_{p}^{\#}(H)=\alpha_{p}^{\#}(G)$.

Proof. As $H$ is embedded $H$-equivariantly, quasi-isometrically in $G$, we have $\alpha_{p}^{\#}(H) \geq \alpha_{p}^{\#}(G)$. To prove the converse inequality, we may assume that $\alpha_{p}^{\#}(H)>0$. Let $S$ be a compact generating subset of $H$. Let $A(h) v=\pi(h) v+b(h)$ be an affine isometric action of $H$ on $L^{p}$, such that for some $\alpha<\alpha_{p}^{\#}(H)$ we have $\|b(h)\|_{p} \geq C|h|_{S}^{\alpha}$, for every $h \in H$. Now, we induce up the action $A$ from $H$ to $G$, as on p. 91 of [6] ${ }^{1}$. The affine space of the induced action is

$$
E:=\left\{f: G \rightarrow L^{p}: f(g h)=A(h)^{-1} f(g), \forall h \in H \text { and almost every } g \in G\right\},
$$

with distance given by $\left\|f_{1}-f_{2}\right\|_{p}^{p}=\sum_{x \in G / H}\left\|f_{1}(x)-f_{2}(x)\right\|_{p}^{p}$. The induced affine isometric action $\tilde{A}$ of $G$ on $E$ is then given by $(\tilde{A}(g)) f\left(g^{\prime}\right)=f\left(g^{-1} g^{\prime}\right)$, for $f \in E$, $g, g^{\prime} \in G$.

A function $\xi_{0} \in E$ is then defined as follows. Let $s_{1}=e, s_{2}, \ldots, s_{n}$ be a set of representatives for the left cosets of $H$ in $G$. Set $\xi_{0}\left(s_{i} h\right)=b\left(h^{-1}\right)$, for $h \in H, i=1, \ldots, n$. Define the 1 -cocycle $\tilde{b}$ on $G$ by $\tilde{b}(g)=\tilde{A}(g) \xi_{0}-\xi_{0}$, for $g \in G$. For an $h \in H$, we then have:

$$
\|\tilde{b}(h)\|_{p}^{p}=\sum_{i=1}^{n}\left\|\xi_{0}\left(h^{-1} s_{i}\right)-\xi_{0}\left(s_{i}\right)\right\|_{p}^{p}=\sum_{i=1}^{n}\left\|\xi_{0}\left(h^{-1} s_{i}\right)\right\|_{p}^{p} \geq\left\|\xi_{0}\left(h^{-1}\right)\right\|_{p}^{p}=\|b(h)\|_{p}^{p} .
$$

Set $K=\max _{1 \leq i \leq n}\left\|\tilde{b}\left(s_{i}\right)\right\|_{p}$. Take $T=S \cup\left\{s_{1}, \ldots, s_{n}\right\}$ as a compact generating set of $G$. For $g \in G$, write $g=s_{i} h$ for $1 \leq i \leq n, h \in H$. Then,

$$
\begin{aligned}
\|\tilde{b}(g)\|_{p} & \geq\|\tilde{b}(h)\|_{p}-K \geq\|b(h)\|_{p}-K \geq C|h|_{S}^{\alpha}-K \geq C|h|_{T}^{\alpha}-K \\
& \geq C\left(|g|_{T}-1\right)^{\alpha}-K \geq C^{\prime}|g|_{T}^{\alpha}-K^{\prime} .
\end{aligned}
$$

So the compression of the 1-cocycle $\tilde{b}$ is at least $\alpha$, hence $\alpha_{p}^{\#}(G) \geq \alpha_{p}^{\#}(H)$.

The following proof uses a construction by S.R. Gal, see page 4 of [12].

THEOREM 3.3. Let $V_{1}$ and $V_{2}$ be closed subspaces of a Hilbert space. Suppose $H$ is a finite index subgroup of $G_{1}$ and $G_{2}$ and suppose there are proper affine isometric actions $\beta_{i}$ (with compression $\alpha_{i}$ ) of each $G_{i}$ on $V_{i}$. Assume that $W<V_{1} \cap V_{2}$ is invariant under

${ }^{1}$ We seize this opportunity to correct a misprint in the definition of the vector $\xi_{0}$ in that construction in p. 91 of [6]. 
the actions $\left(\left.\beta_{i}\right|_{H}\right)$ and moreover that both these (restricted) actions coincide on $W$. Then, $\alpha_{2}^{\#}\left(G_{1} *_{H} G_{2}\right) \geq \frac{\min \left(\alpha_{1}, \alpha_{2}\right)}{2}$. In particular, $\alpha_{2}^{\#}\left(G_{1} *_{H} G_{2}\right) \geq \frac{\alpha_{2}^{\#}(H)}{2}$.

Proof. Following [12], let us build a Hilbert space $W_{\Gamma}$ on which $\Gamma=G_{1} *_{H} G_{2}$ acts affinely and isometrically. Let $\omega$ be a finite alternating sequence of 1's and 2's and suppose $\pi$ is a linear action of $H$ on some Hilbert space denoted $\mathcal{H}_{\omega}$. One can induce up the linear action from $H$ to $G_{i}$, obtaining a Hilbert space

$$
V:=\left\{f: G_{i} \rightarrow \mathcal{H}_{\omega} \mid \forall h \in H, f(g h)=\pi\left(h^{-1}\right) f(g)\right\}
$$

and an orthogonal action $\pi_{i}: G_{i} \rightarrow \mathcal{O}(V)$ defined by $\pi_{i}(g) f\left(g^{\prime}\right)=f\left(g^{-1} g^{\prime}\right)$. The subspace

$$
\left\{f: G_{i} \rightarrow \mathcal{H}_{\omega} \mid \forall h \in H, f(h)=\pi\left(h^{-1}\right) f(1), f_{\mid G_{i} \backslash H}=0\right\},
$$

can be identified with $\mathcal{H}_{\omega}$ by letting an element $f$ correspond to $f(1)$. It is clear that the action $\pi_{i}$ restricted to $H$ coincides with the original linear action $\pi$ via this identification.

So, starting from any linear $H$-action on a Hilbert space $\mathcal{H}_{\omega}$, we can obtain a linear action of say $G_{1}$ on a Hilbert space that can be written as $\mathcal{H}_{\omega} \oplus \mathcal{H}_{1 \omega}$ for some $\mathcal{H}_{1 \omega}$. We can restrict this action to a linear $H$-action on $\mathcal{H}_{1 \omega}$ and we can lift this to an action of $G_{2}$ on a space $\mathcal{H}_{1 \omega} \oplus \mathcal{H}_{21 \omega}$ and so on, repeating the process indefinitely. Here, we will execute this infinite process twice.

The first linear $H$-action on which we apply the process is obtained as follows. As $\beta_{i}(H)(W)=W$ for each $i=1,2$, the restriction to $H$ of $\beta_{1}$, gives naturally a linear $H$-action on $\mathcal{H}_{1}:=V_{1} / W$. The second linear $H$-action is obtained by similarly noting that the restriction to $H$ of $\beta_{2}$ gives a linear $H$-action on $\mathcal{H}_{2}:=V_{2} / W$. We then apply the above process indefinitely.

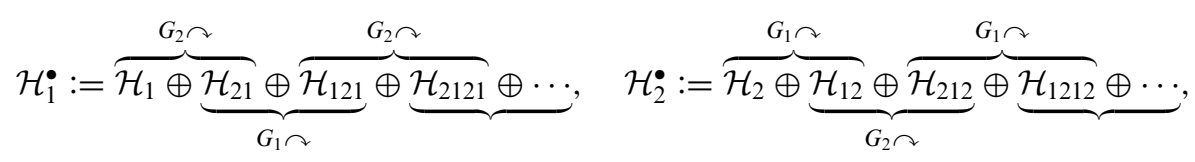

where for $\omega$ a sequence of alternating 1's and 2's, $G_{i}$ acts on $\mathcal{H}_{\omega} \oplus \mathcal{H}_{i \omega}$. Note that there are two $H$-actions on $\mathcal{H}_{1}^{\bullet}$ as $H$ acts on the first term $\mathcal{H}_{1}$. One can verify that both $H$-actions coincide (this fact is also mentioned in [12],page 4). The same is true for $\mathcal{H}_{2}^{\bullet}$.

Denote $\mathcal{H}_{1}^{\circ}=\mathcal{H}_{1}^{\bullet} \ominus \mathcal{H}_{1}$ and similarly, set $\mathcal{H}_{2}^{\circ}=\mathcal{H}_{2}^{\bullet} \ominus \mathcal{H}_{2}$. We denote

$$
W_{\Gamma}=W \oplus \mathcal{H}_{1}^{\bullet} \oplus \mathcal{H}_{2}^{\bullet}=V_{1} \oplus \mathcal{H}_{1}^{\circ} \oplus \mathcal{H}_{2}^{\bullet}=V_{2} \oplus \mathcal{H}_{2}^{\circ} \oplus \mathcal{H}_{1}^{\bullet}
$$

The above formula, which writes $W$ as a direct sum in three distinct ways, shows that both $G_{1}$ and $G_{2}$ act on $W_{\Gamma}$. As mentioned before, the actions coincide on $H$ and so we obtain an affine isometric action of $\Gamma$ on $W_{\Gamma}$. Note that the corresponding 1-cocycle, when restricted to $G_{1}$ (or $G_{2}$ ), coincides with the 1-cocycle of $\beta_{1}$ (or $\beta_{2}$ ).

We inductively define a length function $\psi_{T}: \Gamma \rightarrow \mathbb{N}$ by $\psi_{T}(h)=0$ for all $h \in H$ and $\psi_{T}(\gamma)=\min \left\{\psi_{T}(\eta)+1 \mid \gamma=\eta g\right.$, where $\left.g \in G_{1} \cup G_{2}\right\}$. By applying Proposition 2 in [10] to the Bass-Serre tree of $G_{1} *_{H} G_{2}$, we see that this map is conditionally negative definite and thus the normed square of a 1-cocycle associated to an affine isometric action of $\Gamma$ on a Hilbert space.

Let $\psi_{\Gamma}$ be the conditionally negative definite function associated to the action of $\Gamma$ on $W_{\Gamma}$. We now find the compression of the conditionally negative definite map 
$\psi=\psi_{\Gamma}+\psi_{T}$. First set

$$
M=\max \left\{\left|t_{j}^{i}\right|_{G_{i}}: i=1,2 \text { and } 1 \leq j \leq\left[G_{i}: H\right]\right\},
$$

where $t_{j}^{i}$ are right coset representatives of $H$ in $G_{i}$ such that $t_{1}^{i}=1_{G_{i}}$ for $i=1,2$.

Denote $\alpha=\min \left(\alpha_{1}, \alpha_{2}\right)$ and fix some $\varepsilon>0$ arbitrarily small. Let $\gamma \in \Gamma$ and suppose in normal form $\gamma=g t_{j_{1}}^{i_{1}} \cdots t_{j_{k}}^{i_{k}}$, where $g \in G_{i}$ for some $i=1,2$. Assume first that $\psi_{T}(\gamma) \geq \frac{|\gamma|^{\alpha-\varepsilon}}{M}$. In that case, $\psi(\gamma) \geq \frac{|\gamma|^{\alpha-\varepsilon}}{M}$. Else, we have that $\psi_{T}(\gamma)<\frac{|\gamma|^{\alpha-\varepsilon}}{M}$ and so for all $\gamma \in \Gamma$ such that $|\gamma|$ is sufficiently large, we have

$$
\begin{aligned}
\psi(\gamma) & \geq \psi_{\Gamma}(\gamma)=\|\gamma \cdot 0\|^{2} \\
& \geq\left(\|g \cdot 0\|-\psi_{T}(\gamma) M\right)^{2} \\
& \gtrsim\left(\left(|\gamma|-\psi_{T}(\gamma) M\right)^{\alpha-\varepsilon / 2}-\psi_{T}(\gamma) M\right)^{2} \\
& \geq\left(\left(|\gamma|-|\gamma|^{\alpha-\varepsilon}\right)^{\alpha-\varepsilon / 2}-|\gamma|^{\alpha-\varepsilon}\right)^{2} \\
& \gtrsim|\gamma|^{2 \alpha-\varepsilon},
\end{aligned}
$$

where $\gtrsim$ represents inequality up to a multiplicative constant; we use here that one can always assume, without loss of generality, that the 1-cocycles associated to $\beta_{1}$ and $\beta_{2}$ satisfy $\left\|b_{i}\left(g_{i}\right)\right\| \gtrsim\left|g_{i}\right|^{\alpha-\varepsilon}$ (see Lemma 3.4 in [1]).

So now, by the first case, $\psi(\gamma) \geq|\gamma|^{\alpha-\varepsilon}$ for all $\gamma \in \Gamma$ that are sufficiently large. Hence, we obtain the lower bound $\alpha_{2}^{\#}(\Gamma) \geq \alpha_{2}^{\#}(H) / 2$.

AcKnOwLEDGEMENTS. The authors would like to thank Jacek Brodzki for interesting discussions and the referee for their helpful recommendations and for suggesting a simplier example in Example 2.12. The first author thanks Martin FinnSell for interesting conversations on affine subspaces. The second author thanks Michat Marcinkowski for interesting discussions related to Gal's paper [12]. The first author is sponsored by the EPSRC, grant number EP/I016945/1. The second author is a Marie Curie Intra-European Fellow within the 7th European Community Framework Programme.

\section{REFERENCES}

1. Y. Antolin and D. Dreesen, The Haagerup property is stable under graph products, preprint, 2013.

2. G. N. Arzhantseva, V. S. Guba and M. V. Sapir, Metrics on diagram groups and uniform embeddings in a Hilbert space, Comment. Math. Helv. 81(4) (2006), 911-929.

3. G. Arzhantseva, C. Druţu and M. Sapir, Compression functions of uniform embeddings of groups into Hilbert and Banach spaces, J. Reine Angew. Math. 633 (2009), 213-235.

4. T. Austin, Amenable groups with very poor compression into Lebesgue spaces, Duke Math. J. 159(2) (2011), 187-222.

5. T. Austin, A. Naor and Y. Peres, The wreath product of $\mathbb{Z}$ with $\mathbb{Z}$ has Hilbert compression exponent $\frac{2}{3}$, Proc. Amer. Math. Soc. 137(1) (2009), 85-90.

6. B. Bekka, P. de la Harpe and A. Valette, Kazhdan's property $(T)$, New Mathematical Monographs, vol. 11 (Cambridge University Press, Cambridge, 2008).

7. P.-A. Cherix, M. Cowling, P. Jolissaint, P. Julg and A. Valette, Groups with the Haagerup property, Progress in Mathematics, vol. 197 (Birkhäuser Verlag, Basel, 2001), Gromov's a-Tmenability.

8. Y. Cornulier, Y. Stalder and A. Valette, Proper actions of wreath products and generalizations, Trans. Amer. Math. Soc. 364(6) (2012), 3159-3184. 
9. Y. de Cornulier, R. Tessera and A. Valette, Isometric group actions on Hilbert spaces: Growth of cocycles, Geom. Funct. Anal. 17(3) (2007), 770-792.

10. P. de la Harpe and A. Valette, La propriété $(T)$ de Kazhdan pour les groupes localement compacts (avec un appendice de Marc Burger), Number 175. 1989, With an appendix by M. Burger.

11. D. Dreesen, Hilbert space compression for free products and HNN-extensions, J. Funct. Anal. 261(12) (2011), 3585-3611.

12. Ś. R. Gal, a-T-menability of groups acting on trees, Bull. Austral. Math. Soc. 69(2) (2004), 297-303.

13. E. Guentner and J. Kaminker, Exactness and the Novikov conjecture, Topology 41(2) (2002), 411-418.

14. E. Guentner and J. Kaminker, Exactness and uniform embeddability of discrete groups, J. London Math. Soc. 70(3) (2004), 703-718.

15. E. Hewitt and K. A. Ross, Abstract harmonic analysis. Vol. II: Structure and analysis for compact groups. Analysis on locally compact Abelian groups, Die Grundlehren der mathematischen Wissenschaften, Band 152 (Springer-Verlag, New York, 1970).

16. V. Lafforgue, Un renforcement de la propriété (T), Duke Math. J. 143(3) (2008), 559-602.

17. S. Li, Compression bounds for wreath products, Proc. Amer. Math. Soc. 138(8) (2010), 2701-2714.

18. A. Naor and Y. Peres, Embeddings of discrete groups and the speed of random walks, Int. Math. Res. Not. (2008). doi: 10.1093/imrn/rnn076.

19. Y. Stalder and A. Valette, Wreath products with the integers, proper actions and Hilbert space compression, Geom. Dedicata 124 (2007), 199-211.

20. R. Tessera, Asymptotic isoperimetry on groups and uniform embeddings into Banach spaces, Comment. Math. Helv. 86(3) (2011), 499-535. 